\title{
Emergence of Ixodes scapularis and Borrelia burgdorferi, the Lyme disease vector and agent, in Ohio
}

\section{Peng Wang ${ }^{1}$, Meaghan N. Glowacki ${ }^{1,2}$, Armando E. Hoet ${ }^{2,3}$, Glen R. Needham $^{4}$, Kathleen A. Smith ${ }^{5}$, Richard E. Gary ${ }^{*}$ and Xin Li ${ }^{1 *}$}

${ }^{1}$ Department of Veterinary Biosciences, The Ohio State University, Columbus, OH, USA

${ }^{2}$ Department of Veterinary Preventive Medicine, The Ohio State University, Columbus, OH, USA

${ }^{3}$ Division of Epidemiology, The Ohio State University, Columbus, OH, USA

${ }^{4}$ Department of Entomology, The Ohio State University, Columbus, OH, USA

${ }^{5}$ Ohio Department of Health, Columbus, $\mathrm{OH}$, USA

\section{Edited by:}

Tanja Petnicki-Ocwieja, Tufts

University School of Medicine, USA

Reviewed by:

Peter Kraiczy, University Hospital of

Frankfurt, Germany

Roger W. Stich, University of

Missouri, USA

\section{*Correspondence:}

Richard E. Gary, State Public Health

Entomologist, Ohio Department of

Health, 246 North High Street,

Columbus, OH 43215, USA

e-mail: richard.gary@odh.ohio.gov;

Xin Li, Department of Veterinary

Biosciences, The Ohio State

University, 1900 Coffey Road,

Columbus, OH 43210, USA

e-mail: li.1315@osu.edu
Lyme disease, the most common vector-borne disease in the United States, is caused by a tick-borne infection with Borrelia burgdorferi. Currently, Ohio is considered by the Centers for Disease Control and Prevention (CDC) to be non-endemic for Lyme disease. The low incidence of Lyme disease in this state was largely attributed to the absence of the transmitting vector, Ixodes scapularis, commonly known as the blacklegged tick. However, a tick surveillance program established by Ohio Department of Health indicated that the number of $I$. scapularis in Ohio had increased sharply in recent years, from 0 - 5 ticks per year during 1983-2008 to 15 in 2009, 40 in 2010, and 184 in 2011. During the fall deer hunting season, examination of deer heads submitted to Ohio Department of Agriculture found 29 I. scapularis from 7 counties in 2010 and 1,830 from 25 counties in 2011. As of 2012, the tick had been found in 57 of the 88 counties of Ohio. In addition, all three active stages (larva, nymph, and adult) of $I$. scapularis were found in Tiverton Township of Coshocton County, demonstrating the presence of established tick populations at this central Ohio location. Of 530 nymphal or adult I. scapularis analyzed by quantitative polymerase chain reaction (qPCR), $32(6.1 \%)$ tested positive for the B. burgdorferi flaB gene, ranging from 36 to 390,000 copies per tick. Antibodies to B. burgdorferi antigens were detected in 2 of $10(20 \%)$ field-captured Peromyscus leucopus from Tiverton Township, and in 41 of $355(11.5 \%)$ dogs residing in Ohio. Collectively, these data suggest that the enzootic life cycle of $B$. burgdorferi has become established in Ohio, which poses risk of Lyme disease to people and animals in the area.

Keywords: Lyme disease, Ohio, Borrelia burgdorferi, Ixodes scapularis, Peromyscus leucopus

\section{INTRODUCTION}

Since its initial description in late 1970's (Steere et al., 1977), Lyme disease has been recognized as the most common vectorborne disease in temperate regions of the northern hemisphere (Steere, 2001; Stanek et al., 2012). The disease is caused by spirochetes belonging to the Borrelia burgdorferi sensu lato complex, which is maintained by an enzootic life cycle typically involving Ixodes species ticks and small vertebrate hosts (Piesman and Gern, 2004). In the United States, Lyme disease is highly endemic in two distinct regions, one in the Northeastern states and the other in the Upper Midwestern states (Orloski et al., 2000; Bacon et al., 2008). In these regions, the blacklegged tick Ixodes scapularis is the transmitting vector, and the white-footed mouse Peromyscus leucopus serves as a common reservoir host for the spirochete (Piesman and Gern, 2004). Lyme disease is also reported in the Western United States, where the western blacklegged tick I. pacificus is the transmitting vector and the dusky-footed wood rat Neotoma fuscipes and California kangaroo rat Dipodomys californicus are the main reservoir hosts (Brown and Lane, 1992).
Ohio is situated between the Northeastern and the Upper Midwestern Lyme disease-endemic regions of the US, and it historically had a low incidence of diagnosed Lyme disease. According to CDC data from 2007 to 2012, the annual incidences of Lyme disease in Ohio and the three surrounding states to its west, Michigan, Indiana, and Kentucky, were all $<1$ case per 100,000 people; the annual incidences in the two states to the east of Ohio were higher, ranging 4-8 cases per 100,000 people in West Virginia and 26-37 cases per 100,000 people in Pennsylvania, a highly endemic area. The low incidence of Lyme disease in Ohio was largely attributed to the absence of $I$. scapularis in the area (Dennis et al., 1998; Hoen et al., 2009; Rollend et al., 2013). Here, we showed that since 2009, there had been an exponential increase in the number of I. scapularis found in Ohio. To further assess the risk of Lyme disease in Ohio, we investigated if there were established I. scapularis populations in Ohio, if the ticks were infected with B. burgdorferi, and if white-footed mice and dogs in Ohio had been exposed to $B$. burgdorferi. Our data suggest that the enzootic life cycle of B. burgdorferi has become established in Ohio. 


\section{MATERIALS AND METHODS OHIO DEPARTMENT OF HEALTH TICK SURVEILLANCE PROGRAM}

In 1983, Ohio Department of Health (ODH) began soliciting ticks from the general public, hospitals, physicians, and local health departments in an effort to determine the distribution and dynamics of pathogens in Ohio tick populations. This program of passive tick surveillance for all tick species continued through 2012 and was promoted through media, university extension fact sheets, health department newsletters, presentations and select mailings (Pretzman et al., 1990). Active surveillance to specifically search for I. scapularis began in 1986, which included examination of trapped rodents, flagging vegetation at suspect Lyme disease locations, and examination of deer brought to Ohio Department of Natural Resources (ODNR) deer check stations. Tick collection records and specimens were maintained at ODH. Due to a loss of funding, the ODH tick surveillance program was discontinued in 2013.

\section{EXAMINATION OF DEER HEADS FOR TICKS}

From 2002 to 2011, Ohio Department of Agriculture (ODA) worked with ODNR and ODH to conduct surveillance of chronic wasting disease (CWD) during the fall deer hunting season by examining hunter-harvested deer heads. These deer heads were also examined for ticks. This active surveillance for CWD was discontinued in 2012.

\section{TICK SURVEY IN TIVERTON TOWNSHIP}

From March to November of 2010, ticks were collected from Tiverton Township in Coshocton County, Ohio by two different methods, flagging ticks from vegetation and soliciting ticks from local residents who found and removed them from people or domestic animals. All ticks collected in the area were identified by Glen R. Needham to be either I. scapularis or Dermacentor variabilis based on morphology.

\section{QUANTITATIVE POLYMERASE CHAIN REACTION (qPCR) FOR DETECTION OF B. BURGDORFERI DNA}

DNA was purified from individual or pooled ticks using the DNeasy Blood and Tissue Kit (QIAGEN) following the manufacturer's protocol, and qPCR reactions (25- $\mu$ l each) were set up in 96-well plates using TaqMan Universal PCR Master Mix (Applied Bioscience) and a pair of primers and a TaqMan probe specific for the B. burgdorferi flaB gene (Li et al., 2007). The usage of a sequence-specific fluorescent probe in qPCR analysis not only provides a higher level of specificity than conventional PCR but also allows real-time detection of signals, circumventing the need of analyzing samples by gel electrophoresis. Reactions were run on a MX3005P QPCR system (Agilent Technologies) and data were analyzed using the MxPro QPCR software (Agilent Technologies). For determination of gene copy number, reactions containing 10-fold serial dilutions of DNA with known concentration were included on each plate. This generated a standard curve to extrapolate gene copy numbers from the threshold cycle $(\mathrm{Ct})$ numbers. Given that only $5 \%$ of the total DNA from each tick was analyzed in a reaction, the theoretic limit of detection was 20 copies of B. burgdorferi genome per tick.

\section{RODENT TRAPPING IN TIVERTON TOWNSHIP}

In September of 2010, rodent trapping was carried out in Tiverton Township for two consecutive nights. Sherman live traps (150 at the first night and 180 at the second night) were set using sunflower seeds and peanut butter sandwiches as bait. Captured rodents ( 3 from the first night and 7 from the second night) were euthanized by barbiturate overdose followed by heart exsanguination, and then checked for the presence of ticks. All 10 captured rodents were morphologically identified to be P. leucopus by John Harder, a mammalogist at The Ohio State University. Wildlife procedures were carried out in accordance with the Animal Welfare Act and were approved by $\mathrm{ODH}$ as part of disease surveillance investigation.

\section{ENZYME-LINKED IMMUNOSORBENT ASSAY (ELISA)}

Serum samples were tested for IgM and IgG antibodies against $B$. burgdorferi antigens by enzyme-linked immunosorbent assay (ELISA). ELISA was performed in 96-well flat-bottom Immulon 1B plates (Thermo Scientific) according to a standard protocol (Hornbeck et al., 2001). Briefly, the plates were coated with $100 \mathrm{ng} /$ well whole-cell lysate of B. burgdorferi type strain B31. Serum samples were assayed at a 1:400 dilution. The secondary antibodies, either goat anti-mouse IgM or IgG or goat antidog IgG, were conjugated with horseradish peroxidase (HRP) (Kirkegaard \& Perry Laboratories) and used at a 1:1000 dilution. Signals were developed using the TMB Microwell Peroxidase Substrate System (Kirkegaard \& Perry Laboratories) and measured using a SpectraMax M2 plate reader (Molecular Devices, LLC). For the study of B. burgdorferi seroprevalence in Ohio dogs, the titer of a highly positive serum sample was determined using the serial dilution method. Eight two-fold serial dilutions of this sample as well as eight blank wells were included on each ELISA plate, which were used for normalization to minimize plate-to-plate variation and for generating standard curves to extrapolate titers of unknown samples from the absorbance values.

\section{IMMUNOBLOT ANALYSIS}

Borrelia burgdorferi whole cell lysate was separated on a sodium dodecyl sulfate- $12 \%$ polyacrylamide gel prepared using the MiniProtean Tetra hand cast system and a prep/2-D well comb (BioRad Laboratories), and then transferred to a nitrocellulose membrane. Mouse serum samples were incubated at a 1:100 dilution with the membrane in a Mini-Protean II multiscreen apparatus (Bio-Rad Laboratories). After incubation with HRP-conjugated secondary antibodies, goat anti-mouse IgM or IgG, signals were developed using SuperSignal West Pico Chemiluminescent Substrate (Thermo Scientific) following manufacture's protocol, and captured on X-ray films.

\section{ViraStripe ${ }^{\circledR}$ TEST}

ViraStripe ${ }^{\circledR}$ Lineblot test kits (Viramed) received clearance from the Food and Drug Administration (FDA) in December 2009 for use in clinical diagnosis of human Lyme disease. The IgG Lineblot uses all 10 native $B$. burgdorferi $\mathrm{B} 31$ proteins recommended by the CDC for serological evaluation of human exposure to the Lyme disease spirochete. Serum samples from dogs 
and rodents were analyzed according to the manufacturer's protocol with only one modification - the alkaline phosphatase (AP)conjugated secondary antibodies were goat anti-mouse IgM for the rodent samples and rabbit anti-dog IgG for the dog samples. Both of these conjugates were obtained from the Jackson ImmunoResearch Laboratories. The positive, cut-off, and negative controls were processed as instructed using the anti-human IgG-AP conjugate provided in the kit. Signals were developed using the chromogen/substrate solution provided in the kit, and reactions were stopped when the cut-off control was clearly visible. Based on the CDC recommendations, serum samples that reacted with at least 5 of the 10 proteins above the cut-off intensity were interpreted as positive.

\section{STATISTICAL ANALYSIS}

All statistical and graphic analyses were performed using the software GraphPad Prism 5 for Windows (version 5.0.1). All reported $P$ values are 2 -tailed. $P$ values $<0.05$ are considered statistically significant. The statistical test for each $P$ value is indicated in the text.

\section{RESULTS}

\section{EVIDENCE FOR THE EMERGENCE OF I. SCAPULARIS IN OHIO}

In 1989, a nymph collected in Butler County became the first I. scapularis confirmed in Ohio by the ODH tick surveillance program. The number of $I$. scapularis collected annually remained low from 1989 to 2008, averaging 1.75 (range, 0-5), accounting for well below $1 \%$ of ticks collected. Numbers of $I$. scapularis began to increase after 2008, with 15 in 2009, 40 in 2010, 184 in 2011, and 182 in 2012 (Figure 1). By 2012, I. scapularis ticks accounted for $24.8 \%$ of ticks collected by ODH tick surveillance program. Of the 456 I. scapularis collected 1989-2012, there were $315(69 \%)$ female, 127 (28\%) male, 13 (3\%) nymphs, and 1 larva.

Between 2002 and 2008, no I. scapularis were found from examination of approximately 200-500 deer heads every year during the fall deer hunting season as part of the multi-agency surveillance of chronic waste disease (CWD) in Ohio. Almost all ticks found on deer heads during this period were Dermacentor albipictus, the one-host winter tick. In 2009, deer heads examined for CWD were not examined for ticks. In 2010, 29 I. scapularis were recovered from $12(\sim 6 \%)$ of approximately 200 deer heads examined. In 2011, 1,830 I. scapurlaris were recovered from 96 (17\%) of 560 deer heads examined (Table 1). All 1,859 I. scapularis collected from deer heads were adults, 59\% male and $41 \%$ female. The 29 ticks recovered in 2010 were from 7 counties, whereas the 1,830 ticks recovered in 2011 were from 25 counties (Figure 2). There was no noticeable increase in the number of $D$. albipictus found on deer heads in 2010 and 2011.

Taken together, as of 2012, I. scapularis ticks were found in 57 of the 88 counties in Ohio (Figures 1,2 ). The fact that these surveillance activities had been established for many years, and the increase in I. scapularis (both in numbers and in geographical ranges) was not observed until 2009 provides strong evidence that I. scapularis is an emerging vector in Ohio.

\section{EVIDENCE FOR ESTABLISHED I. SCAPULARIS COLONIES IN TIVERTON TOWNSHIP}

On March 8, 2010, 10 adult I. scapularis were collected by flagging in less than $30 \mathrm{~min}$. Between March and November of 2010, 207 adult and $>200$ larval I. scapularis were flagged from vegetation, and 52 nymphal and 14 adult $I$. scapularis were submitted by local residents who found and removed the ticks from people

\begin{tabular}{|c|c|c|c|c|}
\hline Year & $\begin{array}{l}\text { Number of deer } \\
\text { heads examined } \\
\text { for ticks }\end{array}$ & $\begin{array}{l}\text { Number of deer } \\
\text { heads with } \\
\text { I. scapularis }\end{array}$ & $\begin{array}{c}\text { Number of } \\
\text { I. scapularis } \\
\text { (female/male) }\end{array}$ & $\begin{array}{c}\text { Number of } \\
\text { counties where } \\
\text { I. scapularis found }\end{array}$ \\
\hline 2010 & $\sim 200$ & 12 & $12 / 17$ & 7 \\
\hline 2011 & 560 & 96 & $747 / 1,083$ & 25 \\
\hline
\end{tabular}

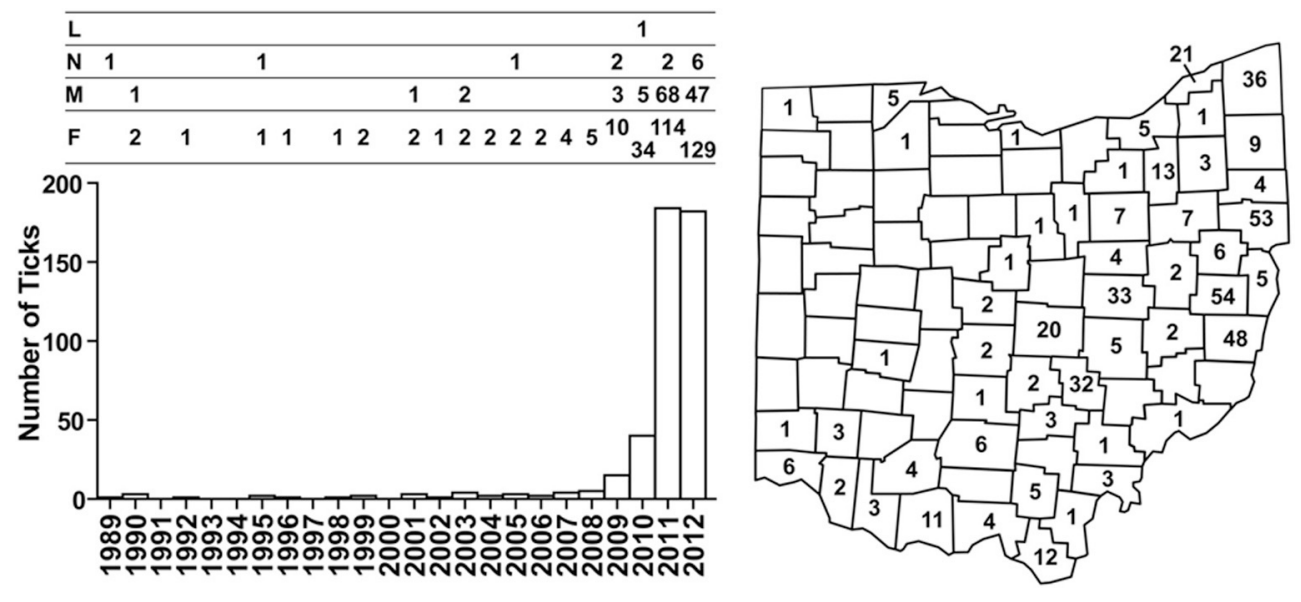

FIGURE 1 | Numbers and distribution of $I$. scapularis collected through the ODH tick surveillance program from 1989 to 2012. Numbers of $I$. scapularis ticks are plotted according to year and the development stages of the ticks are indicated above the bar graph: L, larva; N, nymph; $M$, male, $F$, female. Cumulative numbers of $I$. scapularis found in Ohio counties are indicated on the map. 

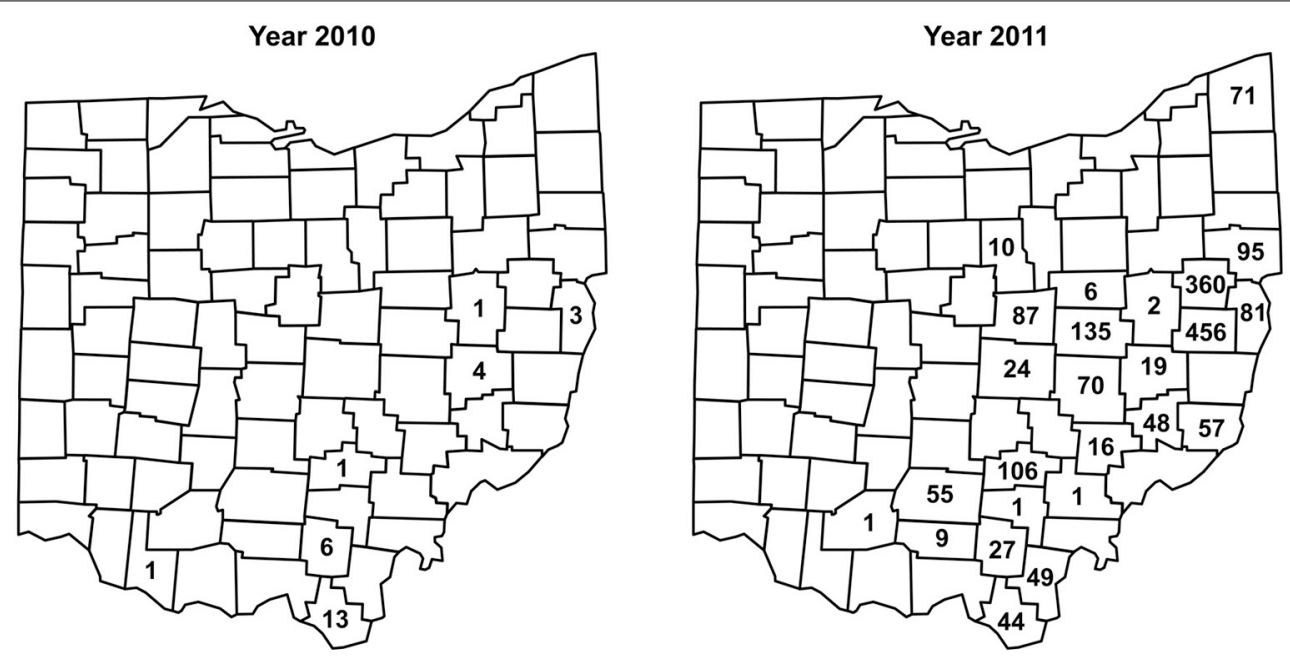

FIGURE 2 | Numbers and distribution of $I$. scapularis found on deer heads through the CWD surveillance program in 2010 and 2011.

Table 2 | Tick survey at Tiverton Township in 2010.

\begin{tabular}{|c|c|c|c|c|}
\hline \multirow[t]{3}{*}{ Month } & \multicolumn{4}{|c|}{ Number of $I$. scapularis } \\
\hline & \multirow{2}{*}{$\frac{\text { Larvae }}{\text { Vegetation }}$} & \multirow{2}{*}{$\begin{array}{c}\text { Nymphs } \\
\text { Host }\end{array}$} & \multicolumn{2}{|c|}{ Adults } \\
\hline & & & Vegetation & Host \\
\hline March & - & - & 27 & - \\
\hline April & - & - & 70 & - \\
\hline May & - & 6 & 43 & 3 \\
\hline June & - & 46 & - & 4 \\
\hline July & $>200$ & - & - & - \\
\hline August & - & - & - & - \\
\hline September & - & - & - & - \\
\hline October & - & - & 15 & 6 \\
\hline November & - & - & 52 & 1 \\
\hline
\end{tabular}

or domestic animals (Table 2). Therefore, all 3 active stages of I. scapularis have been found in Tiverton Township.

\section{PREVALENCE OF B. BURGDORFERI INFECTION IN I. SCAPULARIS COLLECTED IN OHIO}

Nymphal and adult I. scapularis collected from Tiverton Township were analyzed by qPCR to determine the prevalence of B. burgdorferi infection. Larval $I$. scapularis ticks flagged from vegetation were not analyzed because transovarial transmission of B. burgdorferi is rare or nonexistent (Rollend et al., 2013). Initially, the $I$. scapularis ticks were analyzed individually. However, of the 27 adult I. scapularis flagged from vegetation in March 2010, none tested positive, indicating a low prevalence of $B$. burgdorferi infection. Thereafter, some I. scapularis ticks were tested in batches of 2-10 each. Overall, of the 273 I. scapularis tested, 138 were assayed individually and 135 were assayed in batches. Of the 15 I. scapularis samples tested positive for B. burgdorferiflaB DNA, one was a batch of 4 nymphs, one was a batch of 3 nymphs, and all the others were of single I. scapularis. To be conservative in our estimation of the infection rate, in both of these cases where a batch of multiple I. scapularis ticks tested positive, only one was considered positive.

Of the 207 adult $I$. scapularis flagged from vegetation, only $5(2.4 \%)$ tested positive for B. burgdorferi flaB gene (Figure 3). Notably, none of the 140 adult $I$. scapularis collected from vegetation in spring tested positive, whereas 5 of the $67(7.5 \%)$ adults collected in fall tested positive $(P=0.003$, Fisher's exact test). Of the $66 \mathrm{I}$. scapularis collected from people or domestic animals, a total of $10(15.2 \%)$ tested positive. The infection rate was $13.5 \%(7 / 52)$ for the nymphal I. scapularis and $21.4 \%(3 / 14)$ for the adult. In comparison, the infection rate in adult $I$. scapularis flagged from vegetation was significantly lower than that in adults collected from hosts ( $P=0.0095$, Fisher's exact test). Overall, 15 of $273(5.5 \%)$ I. scapularis ticks were infected with B. burgdorferi, and the spirochete burden of infected I. scapularis ranged from 36 to 390,000 per specimen, with a median value of 80 . The tick that had the highest burden was a male that was found crawling off a dog shortly after the removal of a partially engorged female from the animal. Similarly high levels of Borrelia burden in Ixodes species were previously reported by others (Wang et al., 2003; Wilhelmsson et al., 2010).

Although it is not a competent vector for transmitting B. burgdorferi, the dog tick D. variabilis can acquire the spirochete from infected rodents (Soares et al., 2006). Therefore, we also tested 67 adult $D$. variabilis flagged from vegetation in Tiverton Township, and 3 (4.5\%) of them tested positive for B. burgdorferi flaB DNA. This indicated that the infection rate was similarly low in both I. scapularis and D. variabilis found in this area.

Ixodes scapularis from the ODH collections were also tested individually for the presence of $B$. burgdorferi flaB gene. Of the 184 I. scapularis ticks collected through $\mathrm{ODH}$ surveillance in 2011, only 27 were available for DNA testing. Among them, 2 (7.4\%) tested positive, one with 220 and the other with 580 copies of B. burgdorferi genome per tick. Of the 1,830 I. scapularis recovered from CWD deer head surveillance in 2011, 220 (no more than 3 from each deer head) were selected for testing, and 15 


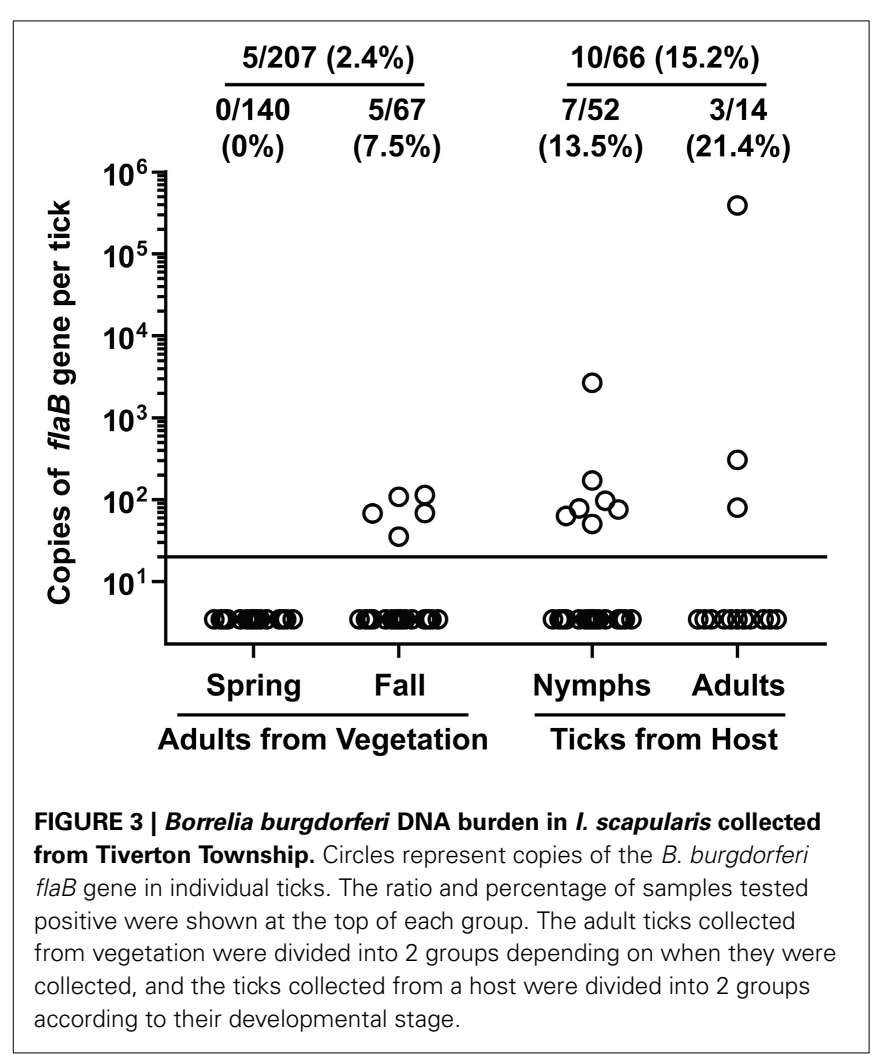

$(6.8 \%)$ tested positive, with the spirochete burden ranging from 111 to 11,800 per ticks. Therefore, the prevalence of $B$. burgdorferi infection in I. scapularis collected from Ohio statewide is similar to that of I. scapularis collected from Tiverton Township.

\section{BORRELIA BURGDORFERI SEROPREVALENCE IN FIELD-CAPTURED P. LEUCOPUS}

We also investigated if the wild rodent population in Tiverton Township had been exposed to B. burgdorferi. In September of 2010, a total of 10 P. leucopus were captured during 2 consecutive nights. Serum samples were tested by ELISA and immunoblot analysis for IgG and IgM antibodies against B. burgdorferi antigens. For comparison, serum samples from laboratory $\mathrm{C} 3 \mathrm{H}$ mice infested by naïve or B. burgdorferi-infected nymphs were included as negative and positive controls, respectively. All 10 P. leucopus serum samples tested negative for IgG antibodies to $B$. burgdorferi, but 2 of them (no. 3 and no. 8 ) had a positive IgM response by both ELISA and immunoblot analysis (Figure 4). It is intriguing that the no. 8 P. leucopus serum sample had much higher reactivity than the no. 3 P. leucopus serum sample in the ELISA assay, but in the immunoblot assay, the results appeared to be reversed, the no. 8 sample yielded much weaker signals than did the no. 3 sample. We repeated the IgM ELISA and immunoblot analyses for all samples to rule out the possibility that we inadvertently switched the samples. One possible explanation for this discrepancy may be that the $B$. burgdorferi component that reacted strongly with the no.8 serum sample in ELISA somehow was not efficiently separated by SDS-PAGE and transferred to the membrane in the immunoblot analysis. Nevertheless, serum samples from infected laboratory mice and the two positive P. leucopus serum samples all reacted very strongly with an approximately $20-\mathrm{kDa}$ Borrelia antigen, which may be the outer surface protein $\mathrm{C}$ (OspC) that is highly expressed during mammalian infection. Thus, we further analyzed these serum samples using the ViraStripe IgG Lineblot, which contains 10 purified native proteins from B. burgdorferi $\mathrm{B} 31$ strain, including the $23-\mathrm{kDa}$ OspC. The result indicated that the common band that reacted with both the laboratory positive controls and the two P. leucopus serum samples was indeed OspC (Figure 4).

The 10 captured $P$. leucopus were examined for ticks. Six mice had a total of 28 ticks attached, which included 23 larval I. scapularis as well as 2 nymphal and 3 larval $D$. variabilis. Only 1 of the 2 D. variabilis nymphs tested positive, which had 267 copies of flaB gene. The D. variabilis nymph that tested positive for $B$. burgdorferi fla $B$ gene was collected from a rodent that tested negative for antibodies against $B$. burgdorferi antigen, suggesting that this dog tick may have acquired the spirochete when taking its first blood meal at the larval stage. One nymphal and 1 larval $D$. variabilis and 12 larval $I$. scapularis were found co-feeding on one of the rodents that tested positive for IgM antibodies to B. burgdorferi, but none of these ticks tested positive for B. burgdorferi DNA.

\section{BORRELIA BURGDORFERI SEROPREVALENCE IN OHIO DOGS}

It has been proposed that seroprevalence in dogs is a good indicator for Lyme disease risk (Bowman et al., 2009; Mead et al., 2011). Therefore, we investigated if there was evidence for B. burgdorferi exposure in Ohio dogs. From June to August of 2011, leftover plasma samples from a total of 355 Ohio dogs that had blood work done at the OSU Veterinary Medical Center were tested by ELISA for IgG antibodies to B. burgdorferi whole-cell lysate. Among them, 197 (55.4\%) were from Franklin County, none were from Coshocton County, and the remaining 158 dogs were from 44 of the other 86 counties in Ohio. Serum samples from 76 healthy greyhound blood donors that had been screened to be free of common vector-borne diseases prior to enrollment and have since been treated with Frontline to prevent vector-borne diseases were included as a control group. The ELISA result indicated that the medium IgG titer was significantly higher in the patient group than in the control group $(P<0.0001$, MannWhitney test) (Figure 5). With an arbitrary cut-off set at the titer of 400 , the seroprevalence was $11.5 \%(41 / 355)$ for the patient group, significantly higher than the $1.3 \%(1 / 76)$ for the control group ( $P=0.0045$, Fisher's exact test). Of the 197 dogs from Franklin County, 26 (13.2\%) tested positive, which is not significantly different from the $9.5 \%$ (15/158) for dogs outside of Franklin County $(P=0.4$, Fisher's exact test $)$.

We next compared the ELISA result with the ViraStripe Lineblot test, an FDA-approved test for diagnosis of human Lyme disease. A total of 71 samples were tested using the ViraStripe IgG Lineblot, which contains 10 purified native B. burgdorferi B31 proteins. Criteria for a positive ViraStripe result are based on the CDC recommendation-reactivity with no less than five of the ten proteins, and each at no less than the cut-off intensity. Notably, these criteria are more stringent and more specific than the criteria that we set for a positive ELISA result-reactivity with B. burgdorferi whole-cell lysate at a titer above 400 . The 71 samples subjected to the ViraStripe test included all 42 ELISA-positive 
IgM Analyses
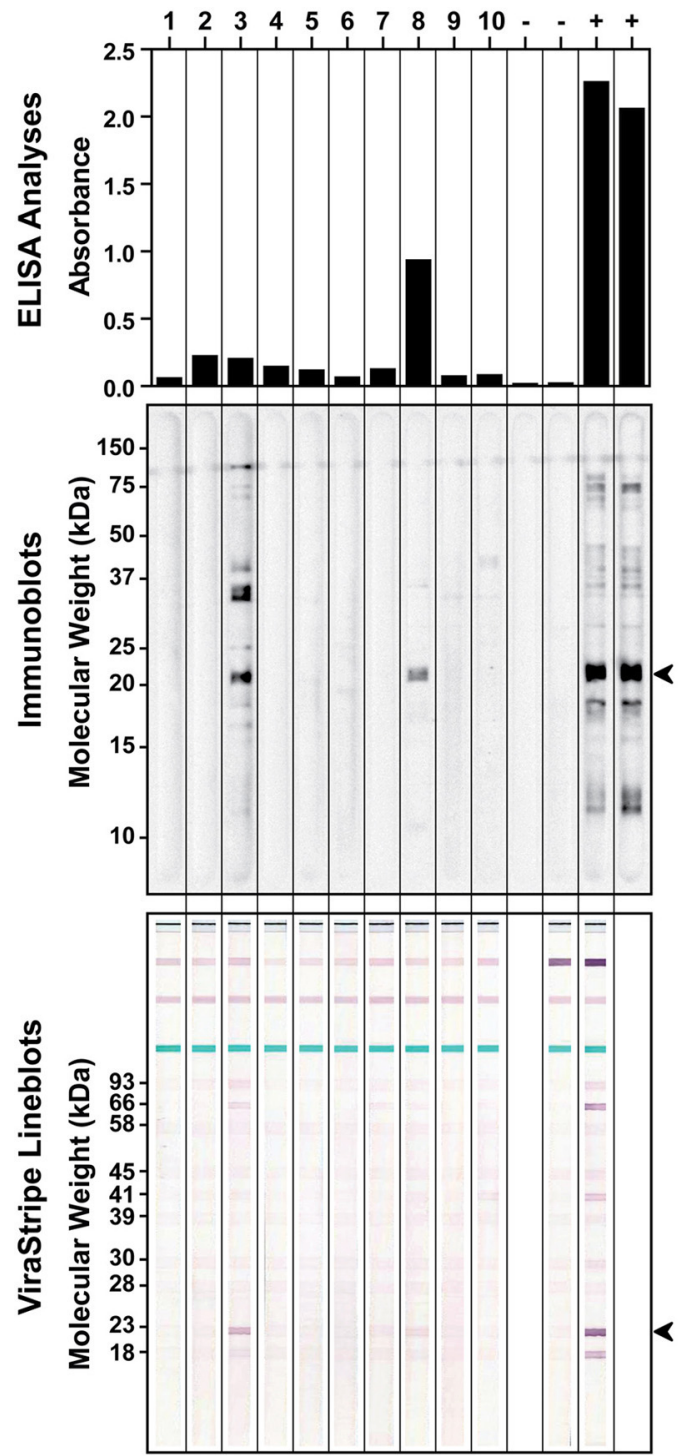

FIGURE 4 | Detection of antibodies to $B$. burgdorferi antigens in

field-captured $\boldsymbol{P}$ leucopus from Tiverton Township. ELISA (top panels) and immunoblot (middle panels) analyses of IgM (left panels) and lgG (right panels) responses to $B$. burgdorferi whole-cell lysate were shown for serum samples
IgG Analyses

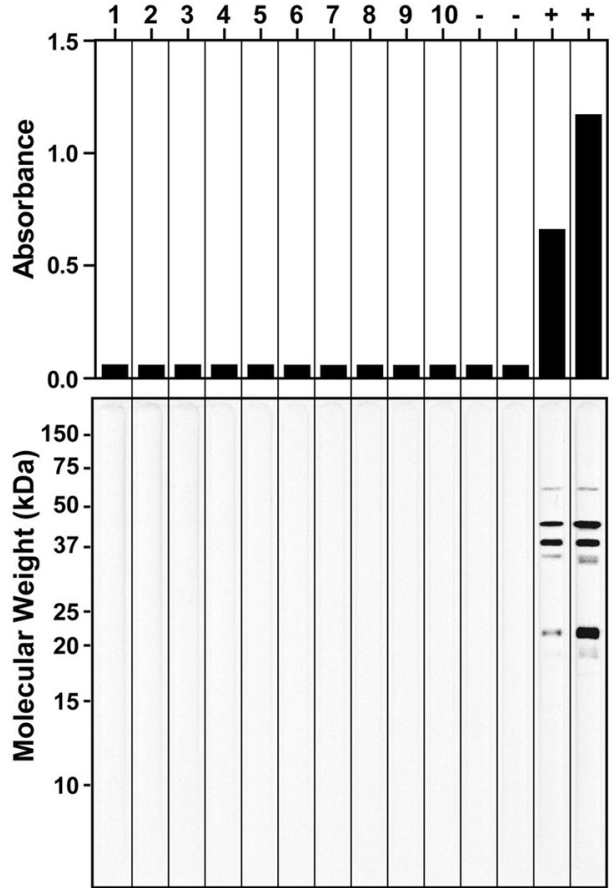

samples (41 patients and 1 greyhound donor) and 29 randomly selected ELISA-negative samples (20 patients and 9 greyhound blood donors). Results are shown in Table 3, and representative blots are shown in Figure 5. Twenty-seven (64\%) of the 42 ELISApositive samples tested positive by ViraStripe, whereas 2 (7\%) of the 29 ELISA-negative samples tested positive by ViraStripe $(P<0.0001$, Fisher's exact test $)$. Moreover, of the ELISA-positive samples, the ones with a higher titer are more likely tested positive by ViraStripe than those with a lower titer. All of the 23 samples that had a titer $\geq 3,122$ tested positive by ViraStripe whereas only 4 of the 19 samples with a titer between 400 and 3104 tested positive by ViraStripe $(P<0.0001$, Fisher's exact test $)$. Therefore, there is from 10 captured $P$. leucopus and 4 laboratory mice that had been infested with naïve (-) or B. burgdorferi-infected ( + ) nymphs. The identity of the $\sim 20-k D a$ protein, indicated by an arrowhead in the $\mathrm{IgM}$ immunoblot, was confirmed to be the 23-kDa OspC by the ViraStripe Lineblot analysis (bottom left panel). 


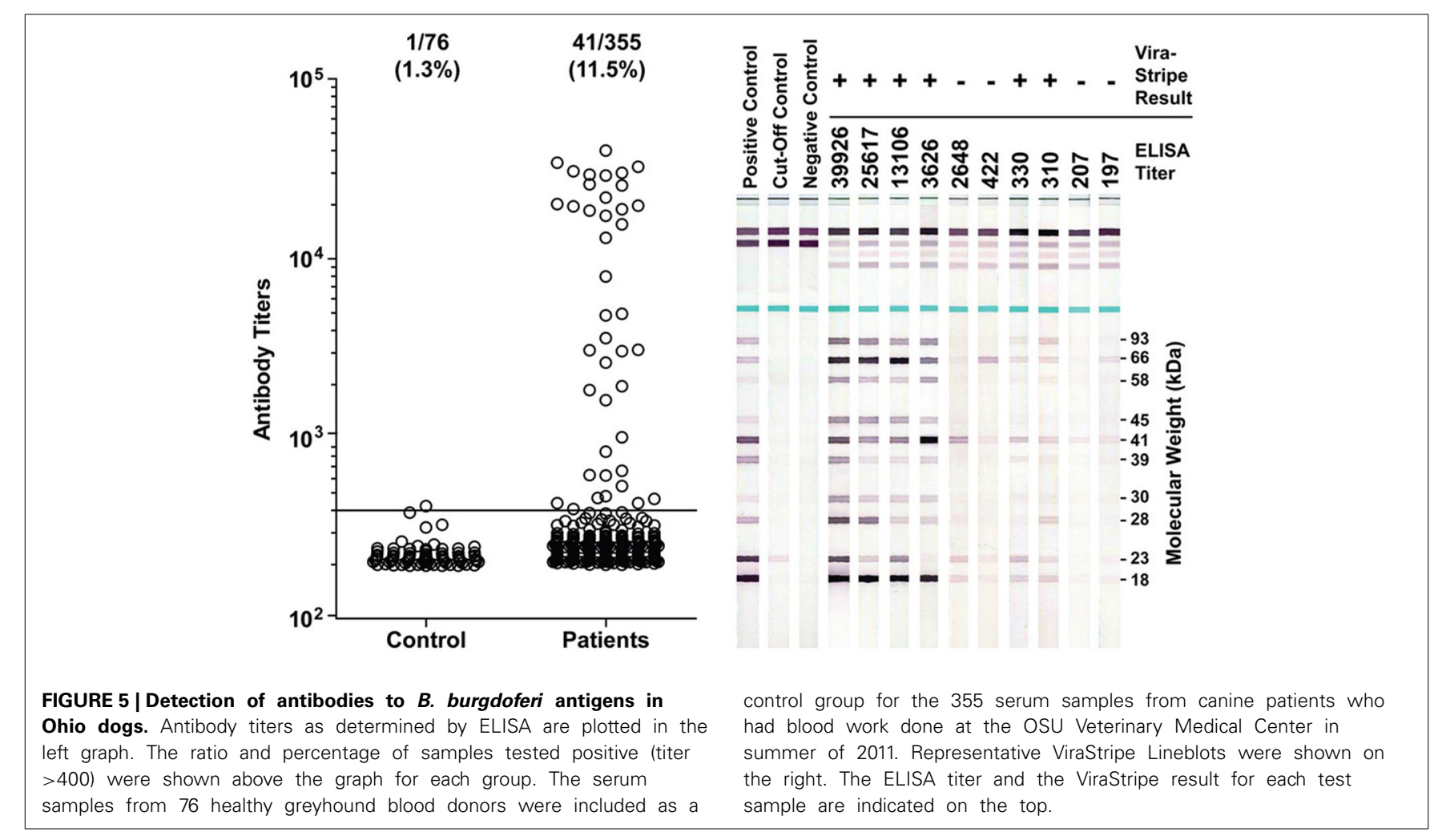

remained low until 2009, and had gone through an exponential increase in the following years. Second, the multi-agency CWD surveillance program that was active 2002-2011 also showed a sharp increase in the number of I. scapularis found on deer heads, from 29 in 2010 to 1830 in 2011. It is important to note that this increase is not due to invigorated surveillance. To the contrary, these data were recorded when both programs were being considered for termination due to decreasing budgets. The CWD monitoring program was terminated after the 2011 season. The ODH tick surveillance program, although active until the end of 2012, was scaled back in that final year, which may explain the plateau in the number of I. scapularis found. It is also important to point out the consistency between these data sets. During 20022008, while the ODH tick surveillance program recorded steady and low numbers of I. scapularis, no such ticks were recovered from deer heads. These data are also consistent with the result from a 2004-2007 survey by others that no nymphs were collected in and around Ohio (Diuk-Wasser et al., 2012). Therefore, the emergence of I. scapularis in Ohio appears to be relatively recent.

Of the 15 I. scapularis that were submitted to ODH in 2009, 3 (20\%) were from Coshocton County. In addition, an Amish family in Tiverton Township of Coshocton County contacted Glen R. Needham regarding a case of un-reported human Lyme disease and sightings of I. scapularis on their property. Glen R. Needham thus surveyed this area in Coshocton County to determine if I. scapularis populations were established. The seasonal activities of larval, nymphal, and adult I. scapularis in Tiverton Township as shown in Table 2 were consistent with those described for other endemic areas in the US (Fish, 1993), with adults being active in spring and fall, nymphs being active in early summer, and larvae being active in summer. Finding all 3 active stages of I. scapularis in Tiverton Township demonstrated the presence of established tick populations in this area, although it did not provide a timeline for the establishment. The low rate of B. burgdorferi infection in the I. scapularis and D. variabilis ticks found in this area and in the field-captured P. leucopus, however, is more consistent with a recent emergence rather than a long-term establishment of the enzootic life cycle in the area.

Given that enzootic life cycle B. burgdorferi in Ohio appears to be nascent, we expect that the number of I. scapularis and the percentage of B. burgdorferi-infected I. scapularis found in this state will continue to increase. However, we do not expect to see further expansion of the range of this tick, because the distribution of I. scapularis found in Ohio so far is, by and large, consistent with the state's deciduous forest range. Given the proximity of these Ohio counties to highly endemic Pennsylvania, one possibility is that the emergence of this tick vector in Ohio simply reflects the continuing expansion of the Northeast Lyme disease-endemic region in the US. Migrating birds and/or deer as well as human activities could play a role in the spreading of the Lyme disease vector and agent. For example, transporting harvested deer from tick-infested areas of nearby Pennsylvania may be a factor because hides are often composted in wooded areas where ticks could detach and begin a "hot-spot." Hunters should be alerted to this possibility so that hides can be properly disposed.

The apparently recent emergence of $I$. scapularis in Ohio is expected to lead to higher incidences of Lyme disease in people and in domestic animals in the future. Of 355 Ohio dog serum samples that were tested by ELISA, $41(11.5 \%)$ had a titer above 400 , and $27(66 \%)$ of these 41 ELISA-positive samples tested positive by an immunoblot analysis using the CDC criteria set for human exposure to the Lyme disease spirochete. Given that 
Table 3 | Comparison of ELISA and ViraStripe analyses of dog serum samples.

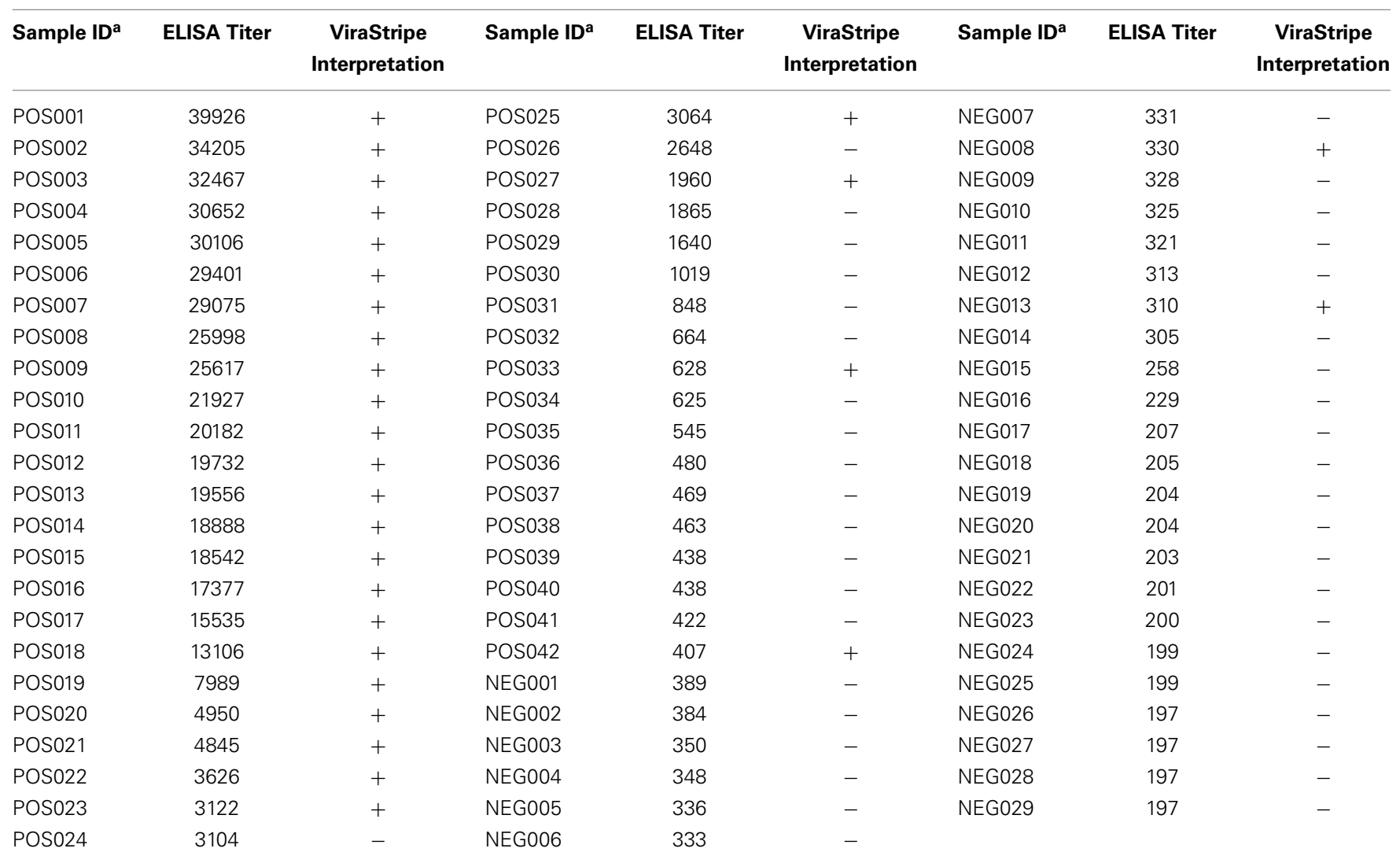

${ }^{a}$ Of the 71 dog serum samples tested by ViraStripe, the 42 that had an ELISA titer above 400 were designated POSO01-042, and the 29 that had an ELISA titer below 400 were designated NEG001-029.

the SNAP 4Dx Plus Test (IDEXX) employs a more stringent criterion for exposure to the Lyme disease spirochete-the presence of antibodies to a specific 26-amino acid residue peptide known as C6 (Liang et al., 1999), not all of the 27 ELISA-positive and ViraStripe-positive samples are expected to test positive by the SNAP 4Dx Plus Test. Also, given that these dogs were seen at a major medical center, the rate of seroprevalence in this group of patients could be higher than that in the general population. According to data published by the Companion Animal Parasite Council website (www.capcvet.org), the percentage of Ohio dogs tested positive for exposure to the Lyme disease spirochete by the SNAP 4Dx Plus Test was $0.39 \%$ (218 of 54,963) for 2011, 0.55\% (250 of 45,376$)$ for $2012,0.56 \%$ for 2013 (543 of 96,016), and $0.62 \%$ (102 of 16,343) for 2014 as of April 23, 2014, showing a significant trend of increase $(P<0.0001$, Chi-square test for trend). According to data available from the $\mathrm{CDC}$, the number of confirmed human Lyme disease cases in Ohio was 21 ( 0.2 per 100,000 persons) for 2010, 36 ( 0.3 per 100,000 persons) for 2011, 49 (0.4 per 100,000 persons) for 2012 , and 74 ( 0.6 per 100,000 persons) for 2013. However, preliminary estimates presented by CDC at the 2013 International Conference on Lyme Borreliosis and Other Tick-Borne Diseases suggest that the actual number of Lyme disease cases may be 10 times higher than the number of reported cases. Given that Ohio was not previously considered endemic for Lyme disease, it is likely that many cases in Ohio might have gone unreported or even undetected. Nevertheless, these numbers of confirmed Lyme disease cases in Ohio, again, indicate a significant trend of increase $(P<0.0001$, Chi-square test for trend). The increased exposure to the Lyme disease agent seen in people and dogs in Ohio further corroborate with our data, and is consistent with a recent emergence of I. scapularis in this state. Healthcare professionals (e.g., physicians, nurse practitioners, and veterinarians) and residents in Ohio should become aware of this emerging tick and its associated risk of disease.

\section{AUTHOR CONTRIBUTIONS}

Glen R. Needham, Kathleen A. Smith, Richard E. Gary, and Xin Li. designed the experiments, Peng Wang and Meaghan N. Glowacki performed laboratory analysis, and all authors contributed to data analysis and interpretation. Peng Wang, Meaghan N. Glowacki and Xin Li wrote the manuscript, and Armando E. Hoet, Glen R. Needham, Kathleen A. Smith, and Richard E. Gary critically revised the manuscript. All authors approved the final manuscript and agreed to be accountable for all aspects of the work in ensuring that questions related to the accuracy or integrity of any part of the work are appropriately investigated and resolved.

\section{ACKNOWLEDGMENTS}

The authors thank Mary Daniels, Jeff Hayes, Tammi Rogers, and Mike Tonkovich for assistance with tick surveillance, David Acosta, Steve Chordas, John Harder and Steve Lonsinger for 
assistance with rodent trapping, and Guillermo Couto and Maxey Wellman for providing dog serum samples. The authors also thank residents at Tiverton Township for their support of the field work. Meaghan N. Glowacki was supported by a T35 Summer Research Fellowship from the National Center for Research Resources. Glen R. Needham was supported by the OSU USDA Hatch Project OHO-01258. This work was supported in part by start-up funds to Xin Li from the College of Veterinary Medicine and the Public Health Preparedness for Infectious Diseases program at The Ohio State University.

\section{REFERENCES}

Bacon, R. M., Kugeler, K. J., Mead, P. S., Centers for Disease, C., and Prevention (2008). Surveillance for Lyme disease-United States, 1992-2006. MMWR. Surveill. Summ. 57, 1-9.

Bowman, D., Little, S. E., Lorentzen, L., Shields, J., Sullivan, M. P., and Carlin, E. P. (2009). Prevalence and geographic distribution of Dirofilaria immitis, Borrelia burgdorferi, Ehrlichia canis, and Anaplasma phagocytophilum in dogs in the United States: results of a national clinic-based serologic survey. Vet. Parasitol. 160, 138-148. doi: 10.1016/j.vetpar.2008.10.093

Brown, R. N., and Lane, R. S. (1992). Lyme disease in California: a novel enzootic transmission cycle of Borrelia burgdorferi. Science 256, 1439-1442. doi: 10.1126/science. 1604318

Dennis, D. T., Nekomoto, T. S., Victor, J. C., Paul, W. S., and Piesman, J. (1998). Reported distribution of Ixodes scapularis and Ixodes pacificus (Acari: Ixodidae) in the United States. J. Med. Entomol. 35, 629-638.

Diuk-Wasser, M. A., Hoen, A. G., Cislo, P., Brinkerhoff, R., Hamer, S. A., Rowland, M., et al. (2012). Human risk of infection with Borrelia burgdorferi, the Lyme disease agent, in eastern United States. Am. J. Trop. Med. Hyg. 86, 320-327. doi: 10.4269/ajtmh.2012.11-0395

Fish, D. (1993). "Population ecology of Ixodes dammini," in Ecology and Environmental Management of Lyme Disease, ed H. S. Ginsberg (New Brunswick, NJ: Rutgers University Press), 1-20.

Hoen, A. G., Margos, G., Bent, S. J., Diuk-Wasser, M. A., Barbour, A., Kurtenbach, K., et al. (2009). Phylogeography of Borrelia burgdorferi in the eastern United States reflects multiple independent Lyme disease emergence events. Proc. Natl. Acad. Sci. U.S.A. 106, 15013-15018. doi: 10.1073/pnas.0903810106

Hornbeck, P., Winston, S. E., and Fuller, S. A. (2001). Enzyme-linked immunosorbent assays (ELISA). Curr. Protoc. Mol. Biol. Chapter 11, Unit 11.12. doi: 10.1002/0471142727.mb1102s15

Li, X., Pal, U., Ramamoorthi, N., Liu, X., Desrosiers, D. C., Eggers, C. H., et al. (2007). The Lyme disease agent Borrelia burgdorferi requires BB0690, a Dps homologue, to persist within ticks. Mol. Microbiol. 63, 694-710. doi: 10.1111/j.1365-2958.2006.05550.x

Liang, F. T., Steere, A. C., Marques, A. R., Johnson, B. J., Miller, J. N., and Philipp, M. T. (1999). Sensitive and specific serodiagnosis of Lyme disease by enzyme-linked immunosorbent assay with a peptide based on an immunodominant conserved region of Borrelia burgdorferi vlsE. J. Clin. Microbiol. 37, 3990-3996.
Mead, P., Goel, R., and Kugeler, K. (2011). Canine serology as adjunct to human Lyme disease surveillance. Emerging Infect. Dis. 17, 1710-1712. doi: $10.3201 / 1709.110210$

Orloski, K. A., Hayes, E. B., Campbell, G. L., and Dennis, D. T. (2000). Surveillance for Lyme disease-United States, 1992-1998. MMWR. Surveill. Summ. 49, 1-11.

Piesman, J., and Gern, L. (2004). Lyme borreliosis in Europe and North America. Parasitology 129(Suppl.), S191-S220. doi: 10.1017/S0031182003004694

Pretzman, C., Daugherty, N., Poetter, K., and Ralph, D. (1990). The distribution and dynamics of Rickettsia in the tick population of Ohio. Ann. N.Y. Acad. Sci. 590, 227-236. doi: 10.1111/j.1749-6632.1990.tb42224.x

Rollend, L., Fish, D., and Childs, J. E. (2013). Transovarial transmission of Borrelia spirochetes by Ixodes scapularis: a summary of the literature and recent observations. Ticks Tick Borne Dis. 4, 46-51. doi: 10.1016/j.ttbdis.2012.06.008

Soares, C. A., Zeidner, N. S., Beard, C. B., Dolan, M. C., Dietrich, G., and Piesman, J. (2006). Kinetics of Borrelia burgdorferi infection in larvae of refractory and competent tick vectors. J. Med. Entomol. 43, 61-67. doi: 10.1603/00222585(2006)043[0061:KOBBII]2.0.CO;2

Stanek, G., Wormser, G. P., Gray, J., and Strle, F. (2012). Lyme borreliosis. Lancet 379, 461-473. doi: 10.1016/S0140-6736(11)60103-7

Steere, A. C. (2001). Lyme disease. N. Engl. J. Med. 345, 115-125. doi: 10.1056/NEJM200107123450207

Steere, A. C., Malawista, S. E., Snydman, D. R., Shope, R. E., Andiman, W. A., Ross, M. R., et al. (1977). Lyme arthritis: an epidemic of oligoarticular arthritis in children and adults in three connecticut communities. Arthritis Rheum. 20, 7-17. doi: 10.1002/art.1780200102

Wang, G., Liveris, D., Brei, B., Wu, H., Falco, R. C., Fish, D., et al. (2003). Real-time PCR for simultaneous detection and quantification of Borrelia burgdorferi in field-collected Ixodes scapularis ticks from the Northeastern United States. Appl. Environ. Microbiol. 69, 4561-4565. doi: 10.1128/AEM.69.8.4561-4565.2003

Wilhelmsson, P., Fryland, L., Borjesson, S., Nordgren, J., Bergstrom, S., Ernerudh, J., et al. (2010). Prevalence and diversity of Borrelia species in ticks that have bitten humans in Sweden. J. Clin. Microbiol. 48, 4169-4176. doi: 10.1128/JCM.01061-10

Conflict of Interest Statement: The authors declare that the research was conducted in the absence of any commercial or financial relationships that could be construed as a potential conflict of interest.

Received: 14 February 2014; accepted: 14 May 2014; published online: 04 June 2014. Citation: Wang P, Glowacki MN, Hoet AE, Needham GR, Smith KA, Gary RE and Li X (2014) Emergence of Ixodes scapularis and Borrelia burgdorferi, the Lyme disease vector and agent, in Ohio. Front. Cell. Infect. Microbiol. 4:70. doi: 10.3389/fcimb. 2014.00070

This article was submitted to the journal Frontiers in Cellular and Infection Microbiology.

Copyright (C) 2014 Wang, Glowacki, Hoet, Needham, Smith, Gary and Li. This is an open-access article distributed under the terms of the Creative Commons Attribution License (CC BY). The use, distribution or reproduction in other forums is permitted, provided the original author(s) or licensor are credited and that the original publication in this journal is cited, in accordance with accepted academic practice. No use, distribution or reproduction is permitted which does not comply with these terms. 